\title{
Stimulus-response compatibility effect in left-right discriminations
}

\author{
LESLIE A. WHITAKER \\ University of Missouri, St. Louis, Missouri 63121
}

\begin{abstract}
Choice response times to visual word and symbol verification tasks were compared for left and right stimulus directions. The results indicated that true statements were responded to more quickly than false statements but that left and right produced equal response times. A strong stimulus-response (S-R) compatibility effect of question (true vs. false) and location of response key (left vs. right side of keyboard) was found. This S-R compatibility effect was based on the concept of verification instead of the left vs. right direction of the stimulus and the corresponding response key per se.
\end{abstract}

When a person is asked to verify a statement about the environment, the speed with which this can be done is a function of a number of variables. Olson and Laxar (1973) found that subjects responded more quickly to right than to left directional statements when the statement included words to indicate directions. Chase and Clark (1971) reported that response times to true statements were faster than response times to false statements. Latencies to symbols vs. words as indicators of direction have been reported by a number of investigators with some inconsistencies as to which stimulus type is preferable (e.g., Dewar, Ells, \& Mundy, 1976; Olson \& Laxar, 1973; Shor, 1971). Finally, the pairing of the stimulus and the response will influence latency and accuracy. This influence of stimulus-response (S-R) pairing on reaction time is known as the $S-R$ compatibility effect (Fitts, 1951). The effects of S-R compatibility on response times to directional stimuli have been reported in several studies (e.g., Craft \& Simon, 1970; Laxar, 1979; Rabbitt, 1967; Wallace, 1972). In general, compatibility is defined as the naturalness of a pairing and is measured by comparing the reaction times to a variety of possible pairings of the same stimuli and responses. The pairing that produces the fastest response time is then defined as the most compatible (Fitts, 1951). At this time, it is not possible to predict the compatibility of many S-R pairings without testing them specifically. Therefore, the effects of direction (left vs. right), statement veridicality (true vs. false), stimulus type (word vs. symbol), and S-R compatibility were investigated in the present study. In this way, it was possible to discover interactions among several variables thought to affect choice response time, as well as to document a compatibility rule for these S-R pairs.

\section{METHOD}

\section{Subjects}

Twenty-three students from the introductory psychology classes at Clarkson College participated as subjects. Eight subjects were women.

\section{Apparatus}

Visual stimuli were presented on a Hazeltine 2000 cathoderay tube (CRT) display controlled by a PDP-11/03. Subjects responded by pressing the $\mathrm{z}$ or the / on the CRT keyboard. Response latency was recorded by the crystal clock in the computer. Latency and accuracy data for each subject were stored on deck tape for subsequent printout and analysis at the campus primary computer facility.

\section{Design}

A repeated-measures design was used. Each subject responded to 20 blocks of eight stimuli. The order of the eight stimuli was randomized within each block. The stimuli were the eight combinations of the following variables: right vs. left, true vs. false, word vs. arrow. For example, the four right-direction stimuli were RIGHT*, ${ }^{*}$ RIGHT, $\rightarrow^{*}$, and ${ }^{*} \rightarrow$. The subject was told that RIGHT* represents the statement "The * is to the right of the word." It is correctly answered, "true." The stimulus $\rightarrow$ * represents the statement "The arrow points to the *." Again, the correct answer is "true."

\section{Procedure}

The subjects were tested individually in a single session; each was seated in front of the CRT and instructed to initiate the trial when he or she was ready. The subject initiated each trial by pressing the space bar with both thumbs. The index finger of each hand was positioned over one of the two response keys. The $z$ (left) and / (right) keys were used as response keys. These were located at the left and right ends of the bottom row on the keyboard, approximately $250 \mathrm{~mm}$ apart. The keys were labeled "true" and "false." Half of the subjects used the lefthand key (z) as true, and half used the right-hand key (/). As soon as the subject initiated the trial, a string of asterisks was presented in the center of the screen. One second later, the asterisk string was replaced with the definitive stimulus. The subject's task was to respond by pressing the appropriate response key as quickly as possible. Choice response time was measured to the nearest $1 \mathrm{msec}$ from the onset of the definitive stimulus to the response. The eight stimuli are shown in Table 1.

Subjects practiced on two sets of the eight stimuli to test their comprehension of the instructions. After these practice trials, the typical reaction time instructions (as fast as possible without making errors) were given, and the 160 test trials began. Three-minute rest periods occurred after each 40 trials.

\section{RESULTS}

Only the latencies of correct responses were analyzed 
Table 1

Stimuli and Responses to Choice RT Task

\begin{tabular}{cccc}
\hline $\begin{array}{c}\text { Symbol } \\
\text { Stimuli }\end{array}$ & Response & Stimuli & Response \\
\hline$\vec{*}^{*}$ & True & RIGHT & True \\
${ }^{*} \rightarrow$ & False & $*$ RIGHT & False \\
${ }_{\leftarrow}^{*}$ & True & $*$ LEFT & True \\
$\leftarrow^{*}$ & False & LEFT ${ }^{*}$ & False \\
\hline
\end{tabular}

Note-The symbol stimuli ask the question, "Does the arrow point at the star?" The word stimuli ask the question, "Is the star on the WORD side?" (For example, RIGHT *: Is the star on the RIGHT side?)

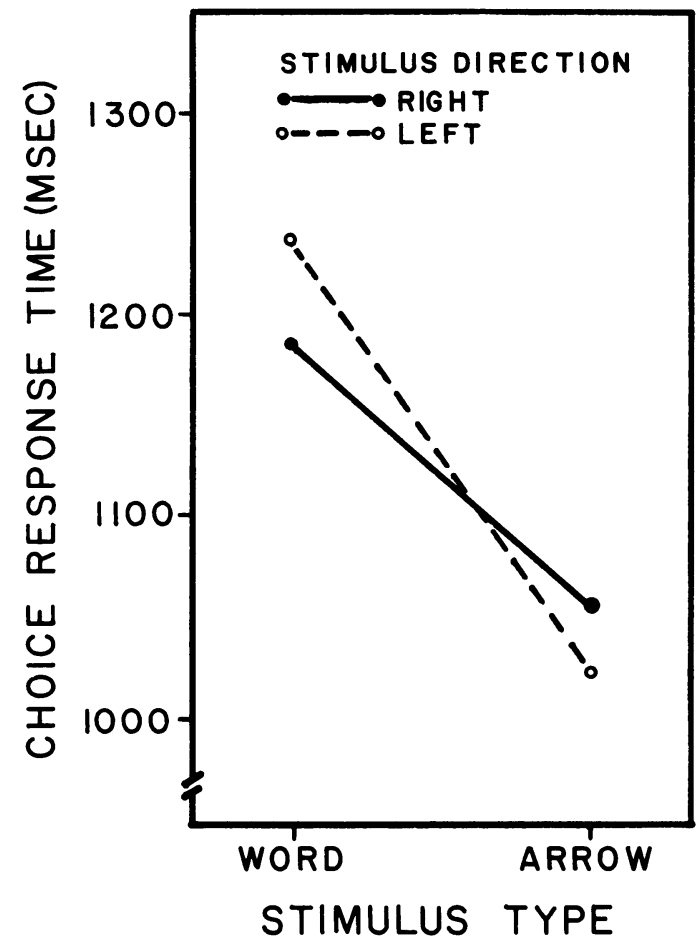

Figure 1. Choice response time as a function of stimulus type and stimulus direction.

in a repeated-measures analysis of variance. The mean response time decreased from $1,252 \mathrm{msec}$ to $1,011 \mathrm{msec}$ from the first to the second half of the 160 choice response time trials. This practice effect was significant $[F(1,19)=49.4, p<.001]$. The mean choice response time for "true" stimuli was 1,064 ; for "false" stimuli it was $1,199 \mathrm{msec}$. This effect of statement veridicality was significant $[F(1,19)=44.2, p<.001]$. The third significant main effect was stimulus type; response times to word stimuli were slower $(1,216 \mathrm{msec})$ than responses to the arrow stimuli $(1,048 \mathrm{msec})$.

Neither left nor right directions produced faster mean responses $(1,136$ and $1,127 \mathrm{msec}$, respectively), but direction did interact with stimulus type. The effect of direction was found in one significant interaction, Stimulus Type by Direction $[F(1,19)=5.44, p<.05]$. This crossover interaction is shown in Figure 1.

Error rates were generally higher for those stimuli that also produced slower response times (see Table 2 for error rates for each stimulus type). Words resulted in more errors than arrows; true and false did not differ, with the exception of the sign "*LEFT." This "true" stimulus was more difficult than any other. Error rate was $16.5 \%$, which is typical of all subjects early and only of one late in the session. In general, first half-session responses had an error rate of $7.4 \%$, second half-session responses had a $3.7 \%$ error rate.

\section{Effects of S-R Compatibility}

The keyboard used to make responses had a left (z) and a right $(/)$ key. The S-R nairings were counterbalanced across subjects, with half of the subjects indicating "true" with the $\mathrm{z}$ key and half with the / key. The effect of the S-R pairings was examined in a compatibility by direction by stimulus type by answer by session half analysis of variance of the choice response times. Two effects involving compatibility were significant. Responses were faster when the direction of the stimulus matched the side of the keyboard holding the "true" response; for example, $\rightarrow *$ and / = true was faster than $\rightarrow^{*}$ and $\mathrm{z}=$ true. This means that the "false" responses were faster when the direction of the stimulus was opposite the side of the keyboard holding the "false" response; for example, ${ }^{*} \rightarrow$ and $\mathrm{z}=$ false was faster than ${ }^{*} \rightarrow$ and $/=$ false. This result was significant $[F(1,18)=24.589, p<.0001]$. The choice response times for compatible and incompatible S-R pairs are listed in Table 3.

In summary, subjects responded to left and right directional signs by indicating whether a word or a symbol statement was true or false. Responses were faster and more accurate for true than for false statements and for symbol than for word statements. Performance did not differ between left and right stimuli. There was a strong S-R compatibility effect of the pairing for statement veridicality and side of response key.

\section{DISCUSSION}

Shorter latencies for true than for false statements have been reported by Olson and Laxar (1973) and were replicated in this experiment. However, those authors found a difference in response times as a function of stimulus direction; right stimuli were responded to more quickly when word stimuli were used. The results of the present experiment require a qualification on this finding. The significant interaction of Direction by Stimulus Type found here indicated a $61-\mathrm{msec}$ advantage for responses to right-direction word stimuli, but a 43-msec advantage for responses to left-direction symbol stimuli. The preferred direction was a function of the stimulus type used. In the research

Table 2

Error Rates in Percentage

\begin{tabular}{lrrrrr}
\hline & \multicolumn{2}{c}{ True } & & \multicolumn{2}{c}{ False } \\
\cline { 2 - 3 } \cline { 5 - 6 } & Left & Right & & Left & Right \\
\hline Word & 16.5 & 6.75 & & 6.00 & 7.00 \\
Arrow & 3.0 & 1.00 & & 2.25 & 1.75 \\
\hline
\end{tabular}

Note-Errors possible in each cell $=400$. 
Table 3

Choice Response Times (RT) in Milliseconds Analyzed for S-R Compatibility

\begin{tabular}{|c|c|c|c|c|c|c|}
\hline & & \multicolumn{3}{|c|}{ Left Key $(Z)$ is True } & \multicolumn{2}{|c|}{ Right Key $(/)$ is True } \\
\hline \multicolumn{2}{|c|}{ Stimulus } & Response & Response Key & RT & Response Key & RT \\
\hline Right Word & $\begin{array}{l}\text { RIGHT * } \\
* \text { RIGHT }\end{array}$ & $\begin{array}{l}\text { True } \\
\text { False }\end{array}$ & $\begin{array}{l}\mathrm{z} \\
\text { l }\end{array}$ & $\begin{array}{l}1270 \\
1307\end{array}$ & $\begin{array}{l}\text { l } \\
\mathrm{z}\end{array}$ & $\begin{array}{r}958 \dagger \\
1207 \dagger\end{array}$ \\
\hline Right Arrow & $\begin{array}{l}\rightarrow^{*} \\
* \rightarrow\end{array}$ & $\begin{array}{l}\text { True } \\
\text { False }\end{array}$ & $\mathrm{z}$ & $\begin{array}{l}1056 \\
1148\end{array}$ & $\begin{array}{l}1 \\
\mathrm{z}\end{array}$ & $\begin{array}{r}983 \dagger \\
1089 \dagger\end{array}$ \\
\hline Left Word & $\begin{array}{l}* \text { LEFT } \\
\text { LEFT * }\end{array}$ & $\begin{array}{l}\text { True } \\
\text { False }\end{array}$ & $\begin{array}{l}z \\
l\end{array}$ & $\begin{array}{l}1129 \dagger \\
1256 \dagger\end{array}$ & $\begin{array}{l}1 \\
\mathrm{z}\end{array}$ & $\begin{array}{l}1216 \\
1386\end{array}$ \\
\hline Left Arrow & $\begin{array}{l}* \leftarrow \\
\leftarrow *\end{array}$ & $\begin{array}{l}\text { True } \\
\text { False }\end{array}$ & $\begin{array}{l}\mathrm{z} \\
\text { l }\end{array}$ & $\begin{array}{l}903 \dagger \\
1121\end{array}$ & $\begin{array}{l}1 \\
\mathrm{z}\end{array}$ & $\begin{array}{c}999 \\
1081 \dagger\end{array}$ \\
\hline
\end{tabular}

†The more compatible response in each case is defined as the faster $S-R$ pairing. In seven of the eight stimuli, the following compatible rule holds: Responding "true" with the same side of the keyboard as the direction of the stimulus is compatible. Conversely, responding "false" with the opposite side of the keyboard is also compatible.

reported here, subjects were tested on both types of stimuli in a single session; Olson and Laxar used word stimuli in one experiment and symbol stimuli in another. It is quite possible that subjects confronted with the larger stimulus set adopted a response set different from that adopted when the stimuli had been divided into two smaller sets. When the stimulus set included word and symbol statements, there was no overall advantage for left or for right directional stimuli.

Word statements resulted in longer latencies than did symbol statements. One reason for this difference might have been the actual discrimination each stimulus type required in order to answer the statement correctly. Corballis and Beale (1976) have described the types of tasks that require that an organism tell left from right. One such task is the one in which a stimulus does not give asymmetric cues while the response requires asymmetrical (left vs. right) responses. In the present experiment, the word statements did not give asymmetric cues, but the symbol statements did. The word statements asked, "Is the * to the WORD side of the stimulus?" The subject had to know the meaning of the words, LEFT and RIGHT, in order to respond correctly. On the other hand, the symbol stimuli did not require that the subject tell left from right in the Corballis and Beale definition because the stimulus gave the asymmetric cue by pointing; "Is the arrow pointing at the *?" does not require that the subject know whether the $*$ is to the left or the right in order to determine the truth of the stimulus statement. However, with the particular response mode required, a left and right response key to indicate true and false, the subject may have coded all responses as left and right. Actually, this would not have to have been the coding. It is conceivable that a human subject with difficulty coding left and right could have responded correctly to the symbol stimuli by using cues in the room and thinking, "The true key is on the side toward the wall and the false is toward the door," or some such code.

In the case of the word stimuli, a subject could not have avoided the left-right coding at some level because the stimulus cue had to be decoded into left and right directions from the words LEFT and RIGHT in order to answer the question about the location of the *. Therefore, the difference in response latencies to word and symbol stimuli could have been a reflection of the complexity of coding necessary to execute the task. However, effects of S-R compatibility were found for both word and symbol stimuli. The S-R compatibility rule found in this experiment was reflected in increased latencies for S-R pairings in which stimuli indicated a true statement about the side of the screen opposite the location of the "true" response key. This held true for false statements as well, in that stimuli making a false statement about the opposite direction from the side of the "false" answer key produced faster responses than did respond- ing with the same-side key. A concept that looks like "oppositeside key equals false and same-side key equals true" appeared in these data. Such a rule should not influence choice response time unless both stimulus types (word and symbol) are being coded for left and right. The faster method of processing a left-right discrimination was found to be the symbol statements. This result has been investigated further in an attempt to determine its generality. Whitaker and Stacey (Note 1) have replicated the faster latencies for arrow stimuli in left-right discriminations, using arrow and word road signs as stimuli.

\section{REFERENCE NOTE}

1. Whitaker, L. A., \& Stacey, S. Response times to left and right directional signs. Manuscript in preparation, 1980.

\section{REFERENCES}

Chase, W. G., \& Clark, H. H. Semantics in the perception of verticality. British Journal of Psychology, 1971, 62, 311-326.

Corballis, M. C., \& Beale, I. L. The psychology of left and right. New York: Wiley, 1976.

Craft, J. L., \& Simon, J. R. Processing symbolic information from a visual display: Interference from an irrelevant directional cue. Journal of Experimental Psychology, 1970, 83, 415-420.

Dewar, R. E., Ells, J. G., \& Mundy, G. Reaction time as an index of traffic sign perception. Human Factors, 1976, 18, 381-392.

FITTS, P. M. Engineering psychology and equipment design. In S. S. Stevens (Ed.), Handbook of experimental psychology. New York: Wiley, 1951.

LAXAR, K. Compatibility and laterality effects in directional information displays. Proceedings of the Human Factors Society, 1979, 433-437.

Olson, G. M., \& Laxar, K. Asymmetrics in processing the terms "right" and "left." Journal of Experimental Psychology, 1973, 100, 284-290.

RABвitT, P. M. Signal discriminability, S-R compatibility, and choice reaction time. Psychonomic Science, 1967, 7, 419-420.

SHor, R. E. Symbol processing speed differences and symbol interference effects in a variety of concept domains. Journal of General Psychology, 1971, 85, 187-205.

Wallace, R. J. Spatial S-R compatibility effects involving kinesthetic cues. Journal of Experimental Psychology, 1972, 93, 163-168.

(Received for publication March 27, 1980.) 